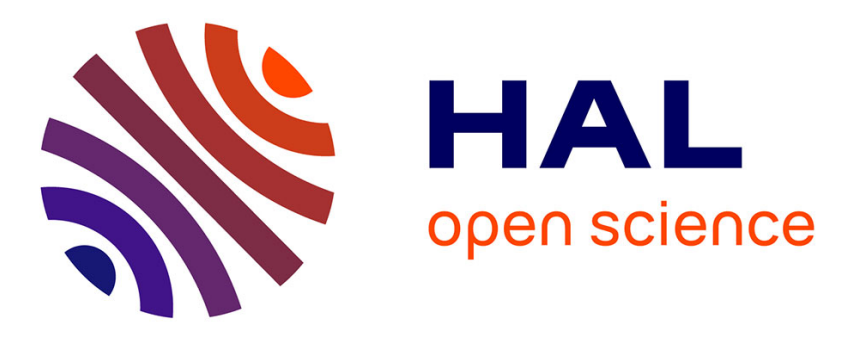

\title{
Source d'ions à ionisation par bombardement électronique ne comportant pas de champ magnétique auxiliaire de source. Etude des caractéristiques du faisceau électronique lorsque les conditions optimales de focalisation sont réalisées
}

Jean Chantreau

\section{To cite this version:}

Jean Chantreau. Source d'ions à ionisation par bombardement électronique ne comportant pas de champ magnétique auxiliaire de source. Etude des caractéristiques du faisceau électronique lorsque les conditions optimales de focalisation sont réalisées. Revue de Physique Appliquée, 1969, 4 (1), pp.13-20. 10.1051/rphysap:019690040101300 . jpa-00242881

\author{
HAL Id: jpa-00242881 \\ https://hal.science/jpa-00242881
}

Submitted on 1 Jan 1969

HAL is a multi-disciplinary open access archive for the deposit and dissemination of scientific research documents, whether they are published or not. The documents may come from teaching and research institutions in France or abroad, or from public or private research centers.
L'archive ouverte pluridisciplinaire HAL, est destinée au dépôt et à la diffusion de documents scientifiques de niveau recherche, publiés ou non, émanant des établissements d'enseignement et de recherche français ou étrangers, des laboratoires publics ou privés. 


\title{
SOURGE D'IONS A IONISATION PAR BOMBARDEMENT ÉLEGTRONIQUE NE GOMPORTANT PAS DE GHAMP MAGNÉTIQUE AUXILIAIRE DE SOURGE ÉTUDE DES GARAGTÉRISTIQUES DU FAISGEAU ÉLEGTRONIQUE LORSQUE LES GONDITIONS OPTIMALES DE FOGALISATION SONT RÉALISÉES
}

\author{
Par JEAN CHANTREAU, \\ Faculté des Sciences de Poitiers, Laboratoire de Spectrométrie de Masse, avenue du Recteur-Pineau, 86-Poitiers.
}

(Reçu le 16 mai 1968, révisé le 30 septembre 1968.)

\begin{abstract}
Résumé. - Un article antérieur [1] traitait de la possibilité d'obtenir un faisceau d'électrons sensiblement afocal et d'épaisseur minimale, par l'adjonction au système accélérateur d'électrons d'un wehnelt, convenablement polarisé. L'étude expérimentale confirmait l'étude théorique pour des tensions accélératrices des électrons comprises entre 100 et $1000 \mathrm{~V}$ et des courants électroniques allant jusqu'à $50 \mu \mathrm{A}$.

Le présent article traite plus particulièrement des caractéristiques du faisceau électronique à la focalisation optimale compte tenu des vitesses initiales des électrons. L'étude théorique a été menée dans l'hypothèse où l'on néglige les phénomènes de charge d'espace. Elle précise les rôles respectifs du wehnelt et des autres électrodes dans la réduction de l'épaisseur du faisceau d'électrons, ainsi que les contributions des diverses régions de la cathode à la formation du faisceau.
\end{abstract}

\begin{abstract}
A mass spectrometer ion source of the Nier type without auxiliary magnetic field has been constructed. In a previous paper [1] we reported experimental and theoretical studies of focalizing conditions for the ionizing electron beam.

The present paper deals with the calculation of the thickness of the electron sheet all along the electron accelerating system for voltages ranging from $100 \mathrm{~V}$ to $1000 \mathrm{~V}$ taking into account the initial velocity of electrons, space-charge being neglected.
\end{abstract}

1. Introduction. - Dans une source d'ions à bombardement électronique de conception classique, on utilise une induction magnétique $\mathbf{B}$ parallèle au faisceau d'électrons pour réaliser dans la chambre d'ionisation un faisceau sensiblement parallèle d'épaisseur aussi faible que possible. La présence de $\mathbf{B}$ perturbe cependant assez profondément les conditions de fonctionnement de la source [2], [3], [4]. Si on s'intéresse en particulier aux diverses causes de discrimination des ions qui ont leur origine au niveau de la source, la présence de $\mathbf{B}$ risque de masquer la contribution qu'apportent à cette discrimination les conditions électriques régnant dans la chambre d'ionisation (charge d'espace, vitesses initiales des ions, pénétration des divers champs accélérateurs dans la chambre, etc.). Il est donc intéressant de réaliser une source d'ions sans induction magnétique auxiliaire, mais pour laquelle il est alors nécessaire d'étudier les conditions optimales à réaliser pour obtenir un faisceau mince d'électrons.

Le canon à électrons d'une source d'ions à bombardement électronique est généralement constitué d'une cathode $\mathrm{K}$, d'une anode A et parfois d'un wehnelt W.
La forme de ces trois électrodes, les distances les séparant et les potentiels qui leur sont appliqués sont très variables d'une réalisation à l'autre.

Lorsqu'il existe, le wehnelt joue le rôle d'une lentille plus ou moins convergente suivant le potentiel auquel il est porté par rapport à la cathode; au contraire, l'anode a une action divergente sur le faisceau. Avec les configurations habituelles, l'action convergente de $\mathrm{W}$ est généralement prépondérante et il en résulte à sa sortie un faisceau convergent dont le cross-over est souvent en deçà de l'anode; le faisceau diverge alors fortement dans la chambre d'ionisation.

Par un réglage correct de la distance $\mathrm{K}-\mathrm{W}$ et de la tension positive intermédiaire appliquée à $\mathrm{W}$, on peut espérer réduire l'action convergente de ce dernier et réaliser un système afocal, pour lequel le faisceau d'électrons sortant de la fente d'anode A ait un angle de divergence nul, toutes choses égales par ailleurs. L'étude expérimentale d'un tel système avait déjà été menée par Vauthier [5] qui constata l'existence d'un minimum d'épaisseur du faisceau électronique, pour une certaine polarisation de $\mathrm{W}$ positive par rapport à $\mathrm{K}$. 
2. La source d'ions. - La source d'ions dont une représentation schématique est donnée figure 1 est de type classique dans sa conception générale. Le

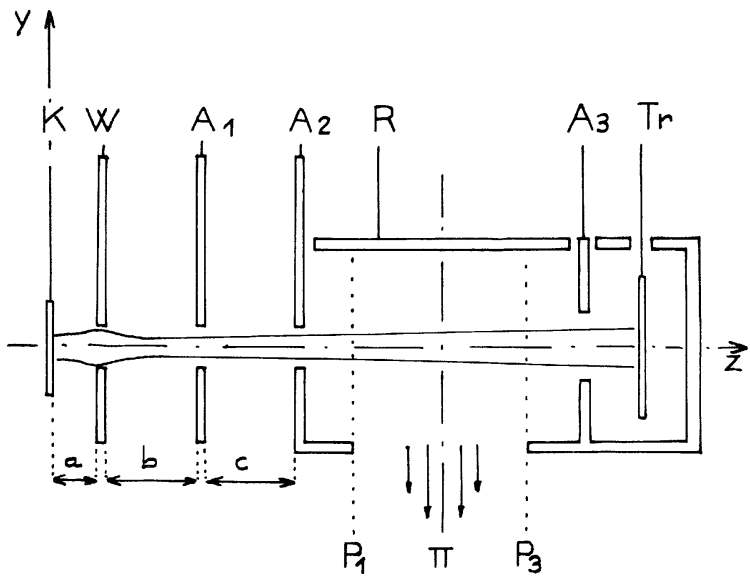

FIG. 1. - Schéma de principe de la source d'ions.

système accélérateur d'électrons est constitué d'une cathode $\mathrm{K}$ (ruban de tungstène), d'un wehnelt $\mathrm{W}$, d'une anode $\mathrm{A}_{1}$. La chambre d'ionisation est délimitée par l'électrode $A_{2}$, le repousseur $R$, l'électrode $A_{3}$ et l'électrode supportant la fente d'extraction des ions. Les électrodes $\mathrm{W}, \mathrm{A}_{1}, \mathrm{~A}_{2}$ ayant des épaisseurs de $0,25 \mathrm{~mm}$ sensiblement égales à la demi-largeur des fentes, on ne peut pas les assimiler à des électrodes minces. Les caractéristiques de construction de la source sont consignées dans le tableau I.

La tension accélératrice $V_{0}$ est appliquée entre $\mathrm{K}$ et $A_{1}$. Les électrodes $A_{2}, A_{3}$ et $R$ et les parois de la chambre, bien qu'isolées électriquement de $A_{1}$, sont également portées au potentiel $V_{0}$. Le wehnelt est porté à une tension intermédiaire réglable :

$$
V_{\mathrm{W}}=\sigma . V_{0}(0<\sigma<1) .
$$

Au-delà de $A_{1}$ et jusqu'en $A_{3}$, les électrons ne sont plus soumis à aucun champ accélérateur. Les dimensions de la fente pratiquée dans $\mathrm{A}_{3}$ sont suffisantes pour que le faisceau d'électrons soit recueilli par la trappe $\mathrm{Tr}$ sans rencontrer les bords de cette fente. Au niveau de $\mathrm{A}_{3}$, les électrons sont soumis au champ faiblement accélérateur appliqué entre $\mathrm{A}_{3}$ et $\mathrm{Tr}$ destiné à supprimer les électrons secondaires créés sur Tr. Pour réduire au maximum les perturbations dans la chambre, dues à cette différence de potentiel, on a particulièrement étudié la géométrie de ces électrodes de façon à rendre très faible la pénétration du champ dans la chambre tout en maintenant entre elles une différence de potentiel de $30 \mathrm{~V}$ suffisante pour neutraliser l'émission secondaire sur $\mathrm{Tr}$.

Le filament est chauffé par une tension alternative de fréquence $1000 \mathrm{~Hz}$. La stabilisation du courant électronique est assurée par régulation du courant de trappe. Cette régulation est très efficace puisque, comme indiqué plus haut, la totalité du courant électronique pénétrant dans la chambre est directement collectée par la trappe.

3. Conditions optimales de focalisation. - L'étude expérimentale de Vauthier reprise dans un esprit différent et complétée par une étude théorique (dans l'hypothèse où l'on néglige la charge d'espace) a fait l'objet d'une publication antérieure [1], dont on rappelle brièvement les points essentiels déjà mentionnés et dont on précise certains points intéressants.

On constate expérimentalement, pour la configuration de source dont les caractéristiques sont définies dans le tableau I, l'existence d'une épaisseur minimale du faisceau correspondant à une valeur de $\sigma=V_{\mathrm{w}} / V_{0}$ égale à 0,12 pour des tensions accélératrices comprises entre $300 \mathrm{~V}$ et $1000 \mathrm{~V}$ (300 V correspondant sensiblement à la limite de sensibilité des substances fluorescentes utilisées pour visualiser le faisceau) et des courants électroniques allant jusqu'à $50 \mu \mathrm{A}$.

La superposition d'une induction magnétique (une centaine de gauss) parallèle au faisceau d'électrons ne réduit pas notablement l'épaisseur de la trace sur l'écran pour les tensions accélératrices comprises entre les limites ci-dessus. On observe par contre une rotation (accompagnée d'une distorsion) de cette trace qui s'incline sur le plan des fentes.

Pour un courant trappe constant, les courants électroniques $I_{\mathrm{W}}, I_{\mathrm{A}_{1}}, I_{\mathrm{A}_{2}}$ passent par un minimum pour la tension wehnelt assurant la trace d'épaisseur minimale; des valeurs typiques des grandeurs électriques

\section{TABLEAU I}

\section{GÉOMÉTRIE DU SYSTÈME ACCÉLÉRATEUR}

$$
\begin{array}{lrl}
a=d_{\mathrm{K}-\mathrm{W}}=2,2 \mathrm{~mm} ; & \text { Fente } \mathrm{W}: L=3 \mathrm{~mm}, l=0,5 \mathrm{~mm} \\
b=d_{\mathrm{W}-\mathrm{A}_{1}}=4-; & \text { Fente } \mathrm{A}_{1}: L=4-l=0,5- \\
c=d_{\mathrm{A}_{1}-\mathrm{A}_{2}}=4-; & \text { Fente } \mathrm{A}_{2}: L=4-l=0,5- \\
\text { Épaisseur : } \mathrm{W}, \mathrm{A}_{1}, \mathrm{~A}_{2}=0,25 \mathrm{~mm} ; & \text { Fente } \mathrm{A}_{3}: L=7-l=1,5- \\
& \text { Cathode (ruban de tungstène) }: L=8-l=0,8-
\end{array}
$$


TABLEAU II

Courants Regueillis Sur Les Diverses ÉleGtrodes

A LA fogalisation optimale pour deUX VAleurs de la tension aGcélératrice $V_{0}$

$$
\begin{aligned}
& \begin{cases}V_{\mathrm{K}}=0 \mathrm{~V} & I_{\mathrm{W}}=120 \quad \mu \mathrm{A}\end{cases} \\
& V_{0}=300 \mathrm{~V} V_{\mathrm{W}}=36 \mathrm{~V} \quad \sigma=0,12 \quad I_{\mathrm{A}_{1}}=4 \mu \mathrm{A} \\
& \begin{array}{ll}
V_{\mathrm{A}_{1}}=V_{\mathrm{A}_{2}}=V_{\mathrm{A}_{3}}=V_{\mathrm{R}}=300 \mathrm{~V} & I_{\mathrm{A}_{2}}=1 \mu \mathrm{A} \\
V_{\mathrm{TR}}=330 \mathrm{~V} & I_{\mathrm{TR}}=10 \mu \mathrm{A}
\end{array} \\
& V_{0}=1000 \mathrm{~V} \begin{cases}V_{\mathrm{K}}=0 \mathrm{~V} & I_{\mathrm{W}}=110 \mu \mathrm{A} \\
V_{\mathrm{W}}=120 \mathrm{~V} \sigma=0,12 & I_{\mathrm{A}_{1}}=3,5 \mu \mathrm{A} \\
V_{\mathrm{A}_{1}}=V_{\mathrm{A}_{2}}=V_{\mathrm{A}_{3}}=V_{\mathrm{R}}=1000 \mathrm{~V} & I_{\mathrm{A}_{2}}=0,5 \mu \mathrm{A} \\
V_{\mathrm{TR}}=1030 \mathrm{~V} & I_{\mathrm{TR}}=10 \mu \mathrm{A}\end{cases}
\end{aligned}
$$

à la focalisation optimale sont consignées dans le tableau II.

L'expérience a montré qu'un défaut d'alignement du filament et des fentes $W, A_{1}$ et $A_{2}$ se traduisait par un décalage les uns par rapport aux autres des minimums de $I_{\mathrm{W}}, I_{\mathrm{A}_{2}}, I_{\mathrm{A}_{2}}$ lorsqu'on faisait varier $V_{\mathrm{W}}$. Une construction mécanique soignée est essentielle.

La focalisation optimale est peu sensible à de légères modifications de la distance $\mathrm{K}-\mathrm{W}$, ce qui s'explique par la distance relativement grande séparant ces deux électrodes. La défocalisation due aux déformations inévitables du filament reste donc toujours faible.

4. Détermination à la cuve rhéographique de la répartition de potentiel sur l'axe. - Pour calculer les trajectoires électroniques paraxiales à travers le système d'électrodes, il suffit de connaître la répartition du potentiel $\Phi(z)$ sur l'axe de symétrie. Il n'a pas été possible de trouver une expression analytique simple de cette répartition, la géométrie de la source différant trop des modèles pour lesquels on sait la calculer.

Pour résoudre le problème tridimensionnel qui nous était posé, on a utilisé la méthode de la cuve rhéographique. On a été amené à réaliser un modèle en laiton à l'échelle $40 / 1$, qui reproduit exactement les formes des électrodes, leurs épaisseurs, ainsi que les longueurs des fentes. Il existe par contre une différence entre la largeur des fentes $W, A_{1}$ et $A_{2}$ réalisée sur la source réelle $(l=0,5 \mathrm{~mm})$ et la largeur de fente du modèle rhéographique $(l=0,4 \mathrm{~mm})$.

Le modèle, de dimensions assez importantes, a été étudié en cuve profonde et les relevés de potentiel ont servi de base aux calculs. Les seules difficultés expérimentales de ces mesures ont été provoquées par la faible impédance électrique (quelques ohms) d'un modèle aussi grand (ce qui a nécessité des aménagements à l'installation d'alimentation $400 \mathrm{~Hz}$ de la cuve) et par la faible dimension de la cathode vis-à-vis des autres électrodes entraînant l'apparition d'impédances de contact importantes dont il a fallu tenir compte.

Un premier relevé expérimental dans tout le plan principal a permis de vérifier la bonne symétrie des équipotentielles de part et d'autre du plan des fentes.

On a ensuite relevé les potentiels sur l'axe pour un certain nombre de valeurs de $V_{\mathrm{w}}$ de part et d'autre de $\sigma=0,12$ correspondant à la focalisation optimale qui avait été obtenue expérimentalement.

La figure 2 représente les variations de $\Phi(z)$ et de sa dérivée $\Phi^{\prime}(z)$ pour $V_{0}=1000 \mathrm{~V}$ et $V_{\mathrm{W}}=160 \mathrm{~V}$.

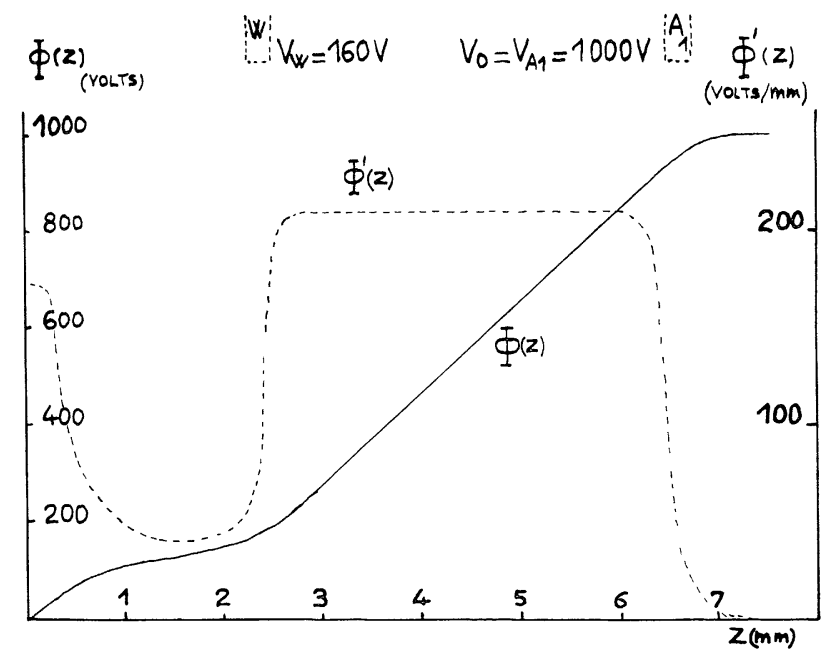

FIG. 2. - Variation de $\Phi(z)$ et de $\Phi^{\prime}(z)$ à la focalisation optimale.

5. Etude des trajectoires à la focalisation optima'e. - L'étude théorique de la publication [1] avait confirmé l'existence d'une tension wehnelt pour laquelle la focalisation était optimale. La valeur de $\sigma$ correspondante était voisine de 0,16 . La différence entre cette valeur théorique de $\sigma$ et la valeur expérimentale $(\sigma=0,12)$ tient à la différence mentionnée plus haut, existant entre la largeur de fente du modèle rhéographique et la largeur de fente de la source réelle. Des considérations de similitude appliquées à la courbe de la publication de Vauthier [5], donnant 
la tension optimale de focalisation en fonction de la distance filament-wehnelt, rendent compte qualitativement et quantitativement du décalage entre les valeurs théorique et expérimentale de $\sigma$.

On a repris plus en détail l'étude théorique des caractéristiques du faisceau pour la valeur particulière de $\sigma(\sigma=0,16)$ qui assure la focalisation optimale théorique.

Des électrons issus de la cathode $K$, suivant une direction faisant l'angle $\theta_{0}$ avec l'axe des $z$, animés d'une vitesse initiale $v_{0}$ possèdent une énergie cinétique qui, en termes de potentiel, a pour expression :

$$
e . \Phi_{0}=\frac{1}{2} \cdot m \cdot v_{0}^{2}
$$

$e$, charge de l'électron, $m$ sa masse.

La trajectoire paraxiale d'un électron pour un système à symétrie plane est alors régie par l'équation ci-dessous [6] :

$2\left[\Phi(z)+\Phi_{\mathrm{i}}\right] \cdot y^{\prime \prime}+\Phi^{\prime}(z) \cdot y^{\prime}+\Phi^{\prime \prime}(z) \cdot y=0$

où :

$$
\Phi_{\mathrm{i}}=\Phi_{0} \cdot \cos ^{2} \theta_{0}
$$

$\Phi(z), \Phi^{\prime}(z), \Phi^{\prime \prime}(z)$ représentent la répartition $\mathrm{du}$ potentiel sur l'axe et ses dérivées successives par rapport à $z$.

Les conditions initiales dépendent des valeurs que l'on fixe pour $\Phi_{0}, Y_{\mathrm{i}}, Y_{\mathrm{i}}^{\prime}=\operatorname{tg} \theta_{0}$.

Les conditions aux limites sont implicitement contenues dans les valeurs de $\Phi(z)$ déduites du relevé expérimental à la cuve.

On a admis la validité de l'équation (2) bien que les fentes des électrodes soient de longueur finie (leur longueur est de l'ordre de dix fois leur largeur).

En dépit des angles $\theta_{0}$ importants des trajectoires issues de $\mathrm{K}$, l'approximation paraxiale est justifiée, car le champ régnant au niveau de $\mathrm{K}$ est élevé et les trajectoires (qui sont d'ailleurs paraboliques si l'on admet - ce qui est justifié - un champ uniforme au voisinage immédiat de $\mathrm{K}$ ) sont très rapidement rabattues sur l'axe et les angles deviennent rapidement très faibles.

Par contre, le calcul des trajectoires extrêmes occultées par les bords de W utilisant l'équation paraxiale n'est qu'approché. Les trajectoires extrêmes réellement suivies par les électrons convergent plus rapidement que celles calculées dans l'approximation gaussienne. Le schéma paraxial a cependant été retenu parce qu'il permet, l'équation (2) étant linéaire, de composer simplement les trajectoires, à partir de la seule répartition axiale, donnant ainsi un ordre de grandeur de l'épaisseur du faisceau.

On néglige, enfin, comme indiqué plus haut, les phénomènes de charge d'espace.
L'équation (2) étant linéaire du second ordre, deux solutions particulières indépendantes $y_{1}$ et $y_{2}$ suffiront à définir la solution générale $y_{3}$ :

$$
y_{3}=\alpha \cdot y_{1}+\beta \cdot y_{2} \text {. }
$$

L'équation (2) régit la trajectoire d'un électron issu d'un point $\mathrm{K}_{1}$ d'ordonnée $Y_{\mathrm{K}_{1}}$ sous l'angle $\theta_{0}$ avec l'énergie initiale $\Phi_{0}$ si l'on fixe les valeurs initiales suivantes :

$$
Y_{i_{3}}=Y_{\mathrm{K}_{1}} ; \quad Y_{\mathrm{i}_{3}}^{\prime}=\operatorname{tg} \theta_{0} ; \quad \Phi_{i_{3}}=\Phi_{0} \cdot \cos ^{2} \theta_{0} .
$$

Mais (2) est la combinaison linéaire de deux trajectoires que nous appellerons trajectoires élémentaires :

- Une trajectoire élémentaire dite de type I issue de $Y_{\mathrm{K}}=0$ sous l'angle $\theta_{0}$ avec l'énergie initiale $\Phi_{\mathbf{0}}$ $\left(Y_{i_{1}}=0, Y_{i_{1}}^{\prime}=\operatorname{tg} \theta_{0}, \Phi_{i_{1}}=\Phi_{0} \cdot \cos ^{2} \theta_{0}\right)$

- Une trajectoire élémentaire dite de type II issue du point $K_{1}$ suivant un angle nul, avec l'énergie initiale $\Phi_{i_{2}}=\Phi_{i_{1}}\left(Y_{i_{2}}=Y_{\mathrm{K}_{1}}, Y_{i_{2}}^{\prime}=0, \Phi_{i_{2}}=\Phi_{i_{1}}\right)$.

Avec ces conditions initiales, il vient : $\alpha=\beta=1$.

Une trajectoire quelconque issue d'un point de $\mathrm{K}$ est donc la somme des deux trajectoires élémentaires correspondantes des types I et II.

D'autre part, deux trajectoires élémentaires de type II issues de deux points distincts $\mathrm{K}_{1}$ et $\mathrm{K}_{2}$ sous un angle nul avec la même énergie $\Phi_{\mathbf{i}}\left(Y_{\mathrm{K}_{1}}=C Y_{\mathrm{K}_{2}}\right.$, $Y_{\mathrm{K}_{1}}^{\prime}=Y_{\mathrm{K}_{2}}^{\prime}=0, \Phi_{i_{1}}=\Phi_{i_{2}}$ ) ont leurs ordonnées qui se déduisent l'une de l'autre par une homothétie de rapport $C$.

Finalement, la connaissance des trajectoires élémentaires de type I et des trajectoires élémentaires correspondantes de type II (mais issues d'un seul point de $\mathrm{K}$ ) permet de calculer n'importe quelle trajectoire satisfaisant à des conditions initiales données.

Une dernière conséquence de la linéarité de (2) permet de limiter les calculs des trajectoires à une seule valeur de la tension accélératrice $V_{0}$ (correspondant bien entendu à $\sigma=0,16)$. On ne modifie pas, en effet, la solution, donc l'allure de la trajectoire, si, pour les conditions initiales identiques $Y_{\mathrm{i}}$ et $Y_{\mathrm{i}}^{\prime}$, on multiplie par une même constante tous les potentiels, y compris le potentiel $\Phi_{0}$ associé à l'énergie initiale des électrons. Deux couples de valeurs $\left(V_{0}, \Phi_{i}\right)$ correspondront à la même trajectoire s'ils sont liés par la relation :

$$
\frac{\Phi_{i_{1}}}{\Phi_{i_{2}}}=\frac{V_{01}}{V_{02}}
$$

Par exemple $V_{01}=1000 \mathrm{~V}, \quad \Phi_{i_{1}}=2 \mathrm{~V}$ et $V_{02}=100 \mathrm{~V}, \quad \Phi_{i_{2}}=0,20 \mathrm{eV}$ correspondent à la même trajectoire.

On a donc finalement calculé les trajectoires élémentaires pour la seule valeur $V_{0}=1000 \mathrm{~V}(\sigma=0,16)$ 
TABLEAU III

Trajectoires du type I : $Y_{\mathrm{i}}=0 \quad Y_{\mathrm{i}}^{\prime}=\operatorname{tg} \theta_{0} \quad \Phi_{\mathrm{i}}=\Phi_{0} \cdot \cos ^{2} \theta_{0}$

$\theta_{0}$ (degrés) : $5, \quad 15, \quad 30, \quad 45, \quad 60, \quad 75, \quad 85$

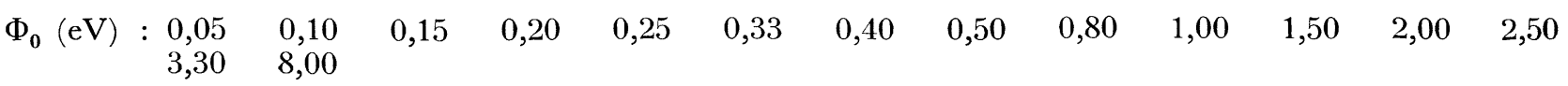

Trajectoires du type II : $Y_{\mathrm{i}}=0,01 \mathrm{~mm} \quad Y_{\mathrm{i}}^{\prime}=0 \quad \Phi_{\mathrm{i}}$

$\begin{array}{rlllllllllllll}\Phi_{\mathrm{i}}(\mathrm{eV}): 0,01 & 0,02 & 0,05 & 0,10 & 0,20 & 0,25 & 0,30 & 0,35 & 0,40 & 0,50 & 0,80 & 1,00 & 1,25 \\ & 1,50 & 2,00 & 2,50 & 3,00 & 3,25 & 3,75 & 4,00 & 4,65 & 5,00 & 6,00 & 7,50 & 8,00 & \end{array}$

et pour les diverses valeurs de $\theta_{0}$ et de $\Phi_{0}$ consignées dans le tableau III ci-dessus.

En toute rigueur, on aurait dû calculer les trajectoires du type II pour autant de valeurs de $\Phi_{\mathrm{i}}$ qu'il y avait de combinaisons simples entre les $\theta_{0}$ et les $\Phi_{0}$ des trajectoires de type I. Cependant, les trajectoires du type II sont très voisines quel que soit $\Phi_{\mathrm{i}}$, ce qui a permis de réduire les valeurs de $\Phi_{\mathrm{i}}$ de 105 à 26 .

5.1. Galgul des trajegtoires élémentaires. Les trajectoires élémentaires des types I et II ont été calculées sur calculatrice numérique IBM 1620. La méthode d'intégration choisie est celle de Gans [7] particulièrement adaptée à l'équation (2).

En effet, en raison, d'une part de la forte valeur du

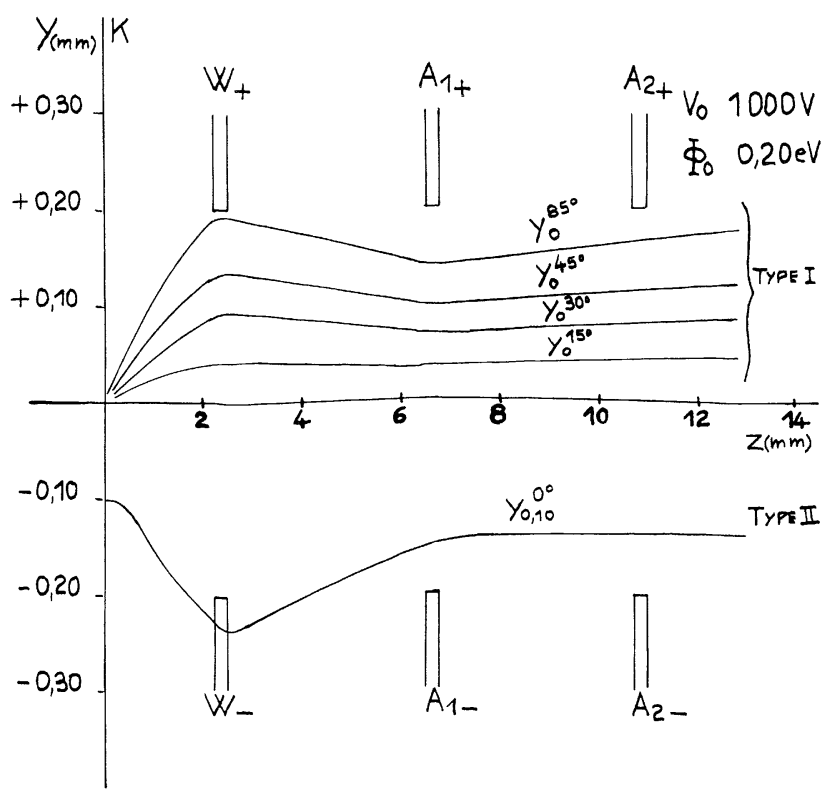

FIG. 3. - Allure des trajectoires élémentaires entre $K$ et $\mathrm{A}_{3}$ pour $V_{0}=V_{\mathrm{A}_{1}}=V_{\mathrm{A}_{2}}=1000 \mathrm{~V}, V_{\mathrm{W}}=160 \mathrm{~V}$ (\# $V_{\mathrm{W}}$ optimal), $\Phi_{0}=0,20 \mathrm{~V}$. champ électrique au niveau de $\mathrm{K}$ et d'autre part de la valeur faible des énergies initiales, les trajectoires partant sous un angle initial $\theta_{0}$ élevé évoluent très rapidement au voisinage de l'origine comme indiqué plus haut. Les dérivées $y^{\prime}$ et $y^{\prime \prime}$ de $y=f(z)$ varient donc beaucoup au voisinage de l'origine et les méthodes générales d'intégration du type Runge-Kutta ne sont applicables que si l'on diminue considérablement la longueur des pas (ce qui augmente en outre corrélativement le nombre de valeurs de $\Phi(z)$ à introduire en données).

Au contraire, la méthode de Gans qui substitue à la fonction $\Phi(z)$ une succession de lignes brisées où le potentiel varie linéairement avec $z$ et qui effectue sur chaque intervalle ainsi déterminé une intégration exacte de l'équation (2) est particulièrement adaptée au calcul des trajectoires au voisinage de l'origine. Quelques essais avec des pas de plus en plus serrés sur plusieurs trajectoires ont permis de vérifier la convergence de la méthode. On a finalement adopté pour $z$ un pas de $0,1 \mathrm{~mm}$, ce qui définit $\Phi(z)$ par 73 valeurs. On a procédé à un lissage des valeurs expérimentales de $\Phi(z)$ avant de les introduire en données; les quelques corrections effectuées sont minimes.

5.1.1. Trajectoires élémentaires de type $I\left(Y_{\mathrm{K}}=0\right.$, $\left.\theta_{0}, \Phi_{0}\right)$. - De telles trajectoires, correspondant à une énergie donnée $\Phi_{0}$, ne franchissent le wehnelt que si l'angle de départ $\theta_{0}$ est inférieur à un certain angle $\left(\theta_{0 \max }\right)_{\mathrm{W}}$ fonction de $\Phi_{0}$.

L'occultation par l'électrode $A_{2}$ se produit de même pour un angle $\left(\theta_{0 \max }\right)_{A_{2}}$ mais supérieur au précédent.

C'est donc en définitive $\mathrm{W}$ qui régit l'occultation des trajectoires de type I.

La figure 4 représente les valeurs de $\theta_{\max }$ en fonction de $\Phi_{0}$ pour $\mathrm{W}$ et $\mathrm{A}_{2}$.

5.1.2. Trajectoires élémentaires de type II $\left(Y_{\mathrm{K}_{1}}, \theta_{0}=0\right.$, $\left.\Phi_{i}\right)$. - Pour ce type de trajectoires, les deux bords $\mathrm{W}_{+}$ et $\mathrm{W}_{-}$(voir fig. 3 ) de $\mathrm{W}$ déterminent pour une énergie $\Phi_{i}$ donnée deux trajectoires extrêmes issues de 


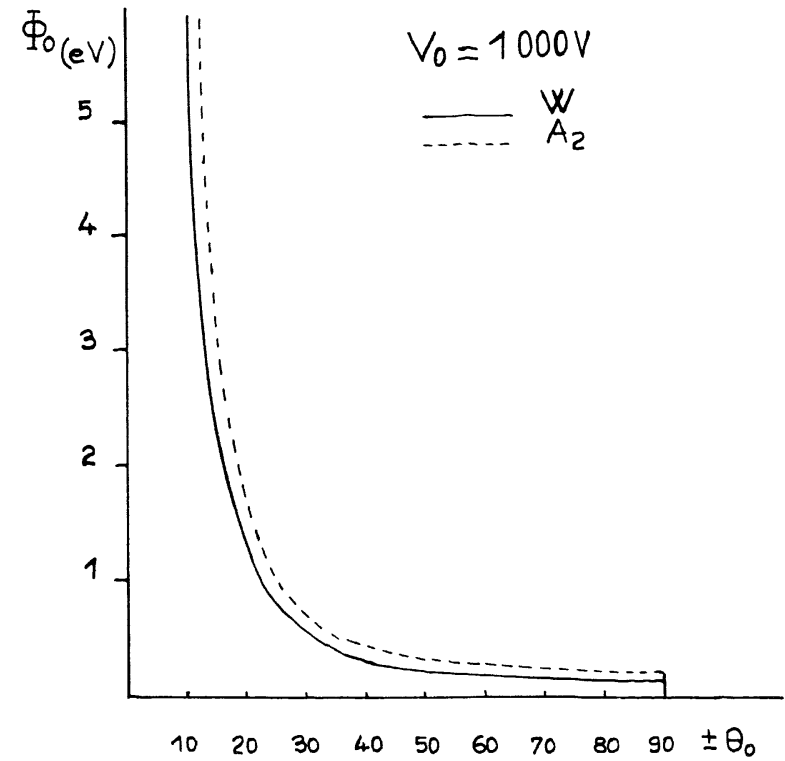

FIG. 4. - Trajectoires de type $I:\left(\theta_{\max }\right)_{\mathrm{W}}$ et $\left(\theta_{0 \max }\right)_{\mathrm{A}_{2}}$ en fonction de $\Phi_{0}$, pour $V_{0}=1000 \mathrm{~V}$.

deux points extrêmes de la cathode $Y_{\mathrm{K}_{1}}$ et $Y_{\mathrm{K}_{\varepsilon}}$ symétriques par rapport à l'axe $\mathrm{z}$ et dépendant de $\Phi_{\mathrm{i}}$. Seuls les points de la cathode situés sur ce segment de longueur $l_{\mathrm{W}}=\left(Y_{\mathrm{K}_{1}}-Y_{\mathbf{K}_{\mathbf{q}}}\right)$ participeront à la formation du faisceau au-delà de W. La largeur utile de $\mathrm{K}$ est donnée en $\mathrm{mm}$, par la formule (5) :

$$
l_{\mathrm{W}}=\frac{0,01 \times 2 s}{Y_{\mathrm{W}_{0,01}^{0}}^{0^{\circ}}\left(\Phi_{\mathrm{i}}\right)}
$$

où $s$ représente la valeur absolue de la demi-largeur de la fente de wehnelt et $Y_{\mathrm{W}_{0,01}^{0}}^{0^{\circ}}\left(\Phi_{\mathrm{i}}\right)$ l'ordonnée en $z=Z_{\mathrm{W}}$ de la trajectoire issue de $Y_{\mathrm{K}}=0,01 \mathrm{~mm}$ normalement à $\mathrm{K}$ avec l'énergie initiale $\Phi_{\mathrm{i}}$. L'occultatation par $\mathrm{A}_{2}$ conduit à une largeur utile de cathode $l_{\mathrm{A}_{2}}$ supérieure à $l_{\mathrm{W}}$.

C'est donc encore $\mathrm{W}$ qui régit l'occultation des trajectoires de type II.

Compte tenu des remarques faites plus haut au sujet des trajectoires de ce type, cette largeur utile de cathode dépend très peu de $\Phi_{i}$ et reste très voisine de $0,1 \mathrm{~mm}$.

5.2. Trajegtoires issues D'Un PoInt QUelconque DE K AVEG L'Énergie initiale $\Phi_{0}$ Sous L'ANgle $\theta_{0}$. De telles trajectoires sont des combinaisons linéaires des trajectoires élémentaires de type I et II. Il faut distinguer leur occultation par le wehnelt et par l'électrode $A_{2}$; l'électrode $A_{1}$ ne participe en effet jamais à l'occultation du faisceau.

6. Résultats. - 6.1. Ocgultation PAR W. - Les ordonnées extrêmes $Y_{\mathrm{K}_{1}}$ et $Y_{\mathrm{K}}$ des points de $\mathrm{K}$ qui contribuent à la formation du faisceau au-delà de $W$ sont pour un angle de départ $\theta_{0}$ et une énergie $\Phi_{0}$, données par les relations :

$$
\begin{aligned}
& Y_{\mathrm{K}_{1}}=\frac{0,01 \times\left[s+Y_{\mathrm{W} 0}^{0_{0}}\left(\Phi_{0}\right)\right]}{Y_{\mathrm{W} 0,01}^{0_{0}^{o}}\left(\Phi_{\mathrm{i}}\right)} \\
& Y_{\mathrm{K}_{2}}=\frac{0,01 \times\left[s-Y_{\mathrm{W} 0}^{\theta_{0}}\left(\Phi_{0}\right)\right]}{Y_{\mathrm{W} 0,01}^{0^{?}}\left(\Phi_{\mathrm{i}}\right)}
\end{aligned}
$$

$Y_{\mathrm{W} 0}^{\theta_{0}}\left(\Phi_{0}\right)$ est l'ordonnée en $z=Z_{\mathrm{W}}$ de la trajectoire (de type $\mathrm{I}$ ) issue de $Y_{\mathrm{K}}=0$ sous l'angle $\theta_{0}$ avec l'énergie initiale $\Phi_{0}$. $Y_{\mathrm{W} 0,01}^{0^{3}}\left(\Phi_{\mathrm{i}}\right)$ est l'ordonnée en $z=Z_{\mathrm{W}}$ de la trajectoire (de type II) issue de $Y_{\mathrm{K}}=0,01 \mathrm{~mm}$, normalement à $\mathrm{K}$ avec l'énergie initiale $\Phi_{\mathrm{i}}=\Phi_{0} \cdot \cos ^{2} \theta_{0}$ (où $\Phi_{0}$ et $\theta_{0}$ ont les valeurs données à la trajectoire de type I ci-dessus).

La largeur utile de cathode correspondante $l_{\mathrm{W}}$ est donc encore égale à :

$$
l_{\mathrm{W}}=\frac{0,01 \times 2 s}{Y_{\mathrm{W} 0,01}^{0^{n}}\left(\Phi_{\mathrm{i}}\right)} .
$$

La largeur utile de cathode due à l'occultation par W conserve donc la même valeur, et pour les mêmes raisons qu'au paragraphe précédent elle évolue peu avec $\Phi_{0}$ et $\theta_{0}$.

Par contre, les ordonnées $Y_{\mathrm{K}_{1}}$ et $Y_{\mathrm{K}_{2}}$ dépendent, par $Y_{\mathrm{W} 0}^{\theta_{0}}\left(\Phi_{0}\right)$, del'angle de départ $\theta_{0}$ et del'énergie initiale $\Phi_{0}$.

Il en résulte un glissement du segment utile $l_{\mathrm{W}}$ au fur et à mesure que $\theta_{0}$ et $\Phi_{0}$ augmentent. Il apparaît d'ailleurs deux segments $l_{\mathrm{W}}$ symétriquement disposés par rapport à l'axe des z, l'un correspondant à l'angle de départ $+\theta_{0}$, l'autre à l'angle de départ $-\theta_{0}$.

La largeur utile de $\mathrm{K}$, pour une énergie et un angle donnés, s'éloigne donc d'autant plus de l'axe $\mathrm{Oz}$ que l'angle de départ et l'énergie initiale des électrons augmentent (fig. 5).

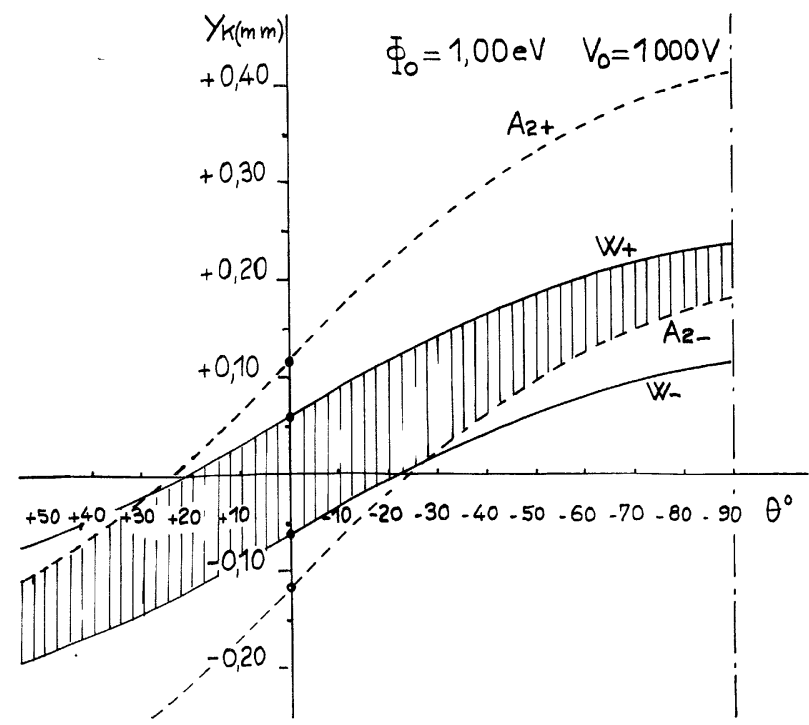

FIG. 5. - Déplacement des largeurs utiles de cathode $l_{\mathrm{W}}$ et $l_{\mathrm{A}_{2}}$ en fonction de $\theta_{0}$ pour $V_{0}=1000 \mathrm{~V}, \Phi_{0}=1 \mathrm{~V}$. La zone hachurée représente la largeur utile résultant de la combinaison des occultations par $A_{2}$ et par W. 
On peut donc conclure que le wehnelt seul n'introduit pas de discrimination des vitesses puisque, quels que soient l'énergie initiale de l'électron et son angle de départ, si les régions concernées de cathode sont différentes, leur largeur reste la même. (Ceci en supposant que la dimension de cathode suivant $Y_{\mathrm{K}}$ est infinie; pour le cas actuel où $Y_{\mathrm{K}}=0,8 \mathrm{~mm}$, toutes les énergies sont cependant identiquement transmises jusqu'à $\Phi_{0}=3,30 \mathrm{~V}$ pour $V_{0}=1000 \mathrm{~V}$, quel que soit l'angle de départ.)

6.2. Ocaultation par $\mathrm{A}_{2}$. - Les ordonnées extrêmes $Y_{\mathbf{K}_{3}}$ et $Y_{\mathbf{K}_{4}}$ des points de $\mathrm{K}$ qui participent à la formation du faisceau au-delà de $\mathrm{A}_{2}$ sont données par les formules $(6)$ et $(7)$ où les $Y_{\mathrm{W}}$ sont remplacés par des $Y_{\mathrm{A}_{2}}$, ordonnées des trajectoires de même type en $z=Z_{\mathrm{A}_{2}}$.

La largeur utile de cathode $l_{\mathrm{A}_{2}}$ délimitée par $\mathrm{A}_{2}$ est donc égale à :

$$
l_{\mathrm{A}_{2}}=\frac{0,01 \times 2 s}{Y_{\mathrm{A}_{2} 0,01}^{0^{\mathrm{o}}}\left(\Phi_{\mathrm{i}}\right)}
$$

$l_{\mathbf{A}_{2}}$ évolue encore très peu avec $\Phi_{i}$.

Par contre, $Y_{\mathrm{W} 0,01}^{0_{0}^{\circ}}\left(\Phi_{\mathrm{i}}\right) \not 2,2 \times Y_{\mathrm{A}_{2} 0,01}^{0^{\prime}}\left(\Phi_{\mathrm{i}}\right)$ (fig. 3$)$ et $l_{\mathrm{A}_{2}}$ est sensiblement double de $l_{\mathrm{W}}$. Mais, d'autre part, $Y_{\mathbf{K}_{3}}$ et $Y_{\mathbf{K}_{4}}$ croissent plus rapidement que $Y_{\mathbf{K}_{\mathbf{1}}}$ et $Y_{\mathbf{K}_{2}}$ avec $\theta_{0}$, en sorte que, pour des valeurs de $\Phi_{0}$ et $\theta_{0}$ suffisamment élevées, l'occultation par le bord $A_{2-}$ de l'électrode $A_{2}$ se produira avant l'occultation par le bord $W_{-}$de l'électrode W ( fig. 5).

L'action combinée de $\mathrm{W}$ et de $\mathrm{A}_{2}$ conduit donc à un chevauchement des zones de transmission correspondant à ces deux électrodes, qui se solde finalement pour des électrons d'énergie initiale donnée par un filtrage des vitesses lorsqu'on atteint des valeurs suffisamment élevées de l'angle de départ ( fig. 5). Il en découle une destruction de l'isotropie d'émission ainsi que de la répartition semi-maxwellienne des vitesses initiales.

L'électrode $\mathrm{A}_{1}$ n'intervient pas dans l'occultation du faisceau électronique, l'occultation par $\mathrm{A}_{2}$ se produisant toujours avant l'occultation par $A_{1}$. Le fait qu'expérimentalement le courant sur $A_{1}$ ne soit pas nul mais supérieur à $A_{2}$ (tableau II) n'est pas en contradiction avec cette conclusion théorique, car le courant recueilli sur $A_{1}$ est dû au courant électronique secondaire issu des électrons primaires occultés par $\mathrm{A}_{2}$ comme il a été possible de le vérifier qualitativement dans des expériences annexes à tension trappe-chambre variable, que nous ne décrirons pas ici.

6.3. Surface utile de CAthode. - Pour $V_{0}=1000 \mathrm{~V}$, la largeur utile de cathode (dont les points participent à la formation du faisceau dans la chambre d'ionisation) reste toujours inférieure à $0,6 \mathrm{~mm}$ pour une largeur effective totale de cathode égale à $0,8 \mathrm{~mm}$ (fig. 6). A noter d'ailleurs que, pour des points de cathode éloignés de l'axe, le filtrage est plus important que celui calculé ici, car le fait que ces points soient au bord de la cathode entraîne une

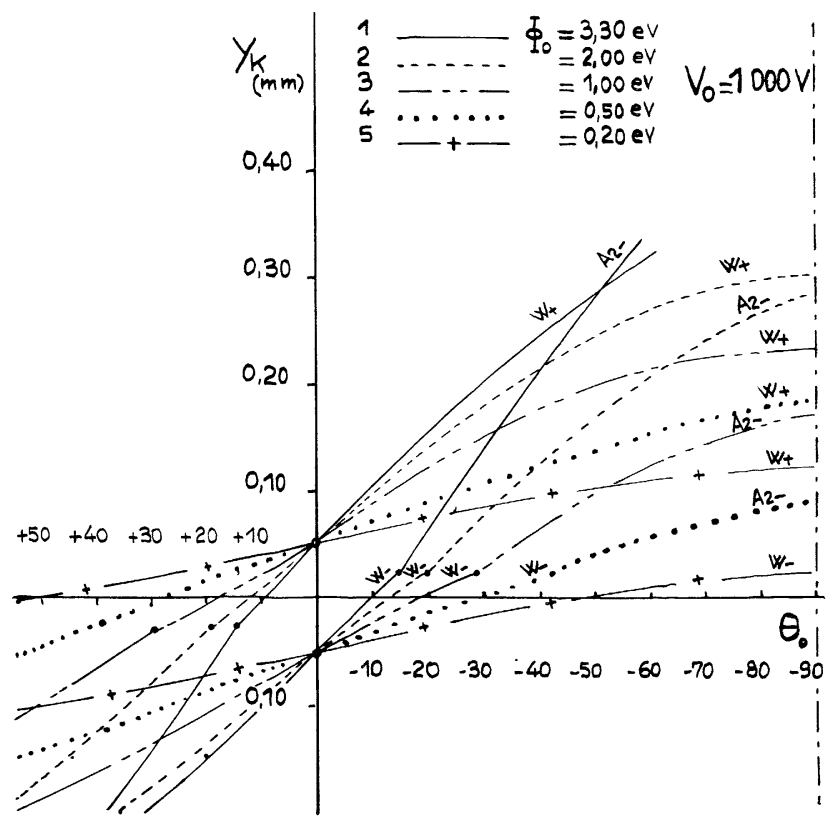

FIG. 6. - Largeurs utiles de cathode résultant des occultations simultanées par $\mathrm{W}$ et $\mathrm{A}_{2}$ pour $V_{\theta}=1000 \mathrm{~V}$ et diverses valeurs de $\Phi_{0}$.

déformation des trajectoires issues de tels points (due à la distorsion des équipotentielles au voisinage des bords de $\mathbf{K}$ ) dont il n'est pas tenu compte dans le présent calcul.

La largeur utile de cathode évolue assez rapidement avec $V_{0}$. En effet, sur la figure 6 , la courbe 1 correspond à : $\Phi_{0}=3,30 \mathrm{~V}, V_{0}=1000 \mathrm{~V}$, mais aussi à : $\Phi_{0}=0,33 \mathrm{~V}, V_{0}=100 \mathrm{~V}$.

De même :

$\begin{array}{lll}\text { Courbe 2 }: \Phi_{0}=0,20 \mathrm{~V} ; & V_{0}=100 \mathrm{~V} \\ \text { Courbe 3 }: \Phi_{0}=0,20 \mathrm{~V} ; & V_{0}=200 \mathrm{~V} \\ \text { Courbe } 4: \Phi_{0}=0,20 \mathrm{~V} ; & V_{0}=400 \mathrm{~V} \\ \text { Courbe 5 : } \Phi_{0}=0,20 \mathrm{~V} ; & V_{0}=1000 \mathrm{~V} .\end{array}$

Donc, si l'on admet (ce qui n'est pas tout à fait exact) que la température de la cathode reste sensiblement constante et correspond à une énergie $\Phi_{0}=0,20 \mathrm{~V}$ quel que soit $V_{0}$, et si l'on néglige les énergies au-dessus de $\Phi_{0}=0,20 \mathrm{~V}$ qui ont des densités de probabilité rapidement très faibles, les résultats ci-dessus permettent de préciser la largeur utile de cathode aux diverses valeurs de $V_{0}$.

$V_{0}=100 \mathrm{~V}$, Largeur utile de cathode : $0,60 \mathrm{~mm}$

$V_{0}=200 \mathrm{~V} \quad-\quad-\quad 0,50 \mathrm{~mm}$

$V_{0}=400 \mathrm{~V} \quad-\quad-\quad-\quad 0,40 \mathrm{~mm}$

$V_{0}=1000 \mathrm{~V} \quad-\quad-\quad 0,25 \mathrm{~mm}$.

6.4. Largeur du faisceau AU-dela de $\mathrm{A}_{2}$. - Les trajectoires extrêmes calculées plus haut permettent de préciser de la même manière la géométrie du faisceau dans la chambre d'ionisation dans l'hypothèse 


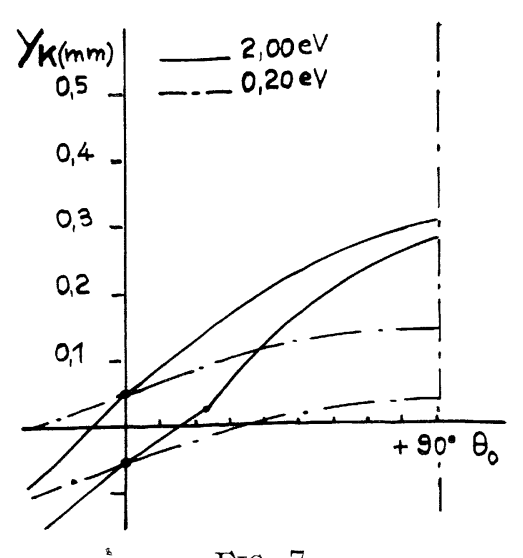

FIG. 7.

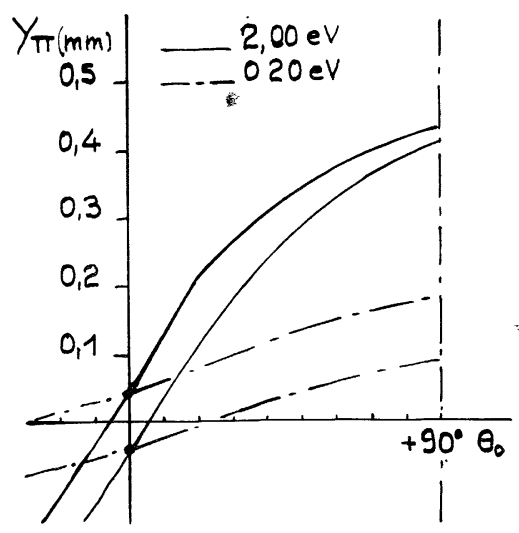

FIG. 8.

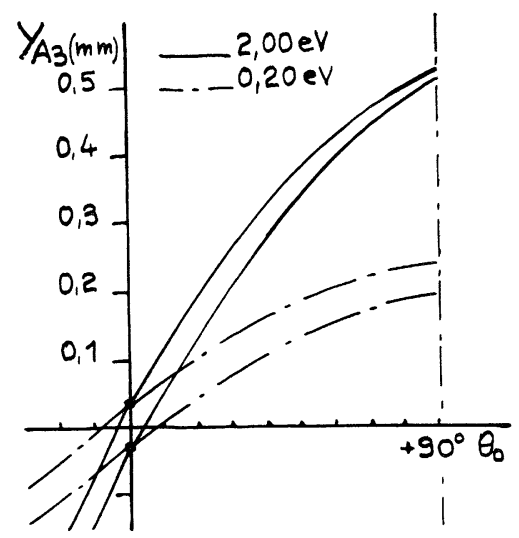

FIG. 9.

FIG. 7 à 9. - Largeur du faisceau électronique dans les plans $\mathrm{K}, \pi, \mathrm{A}_{3}$ ( fig. 1) pour $V_{0}=1000 \mathrm{~V}$ et deux valeurs de $\Phi_{0}$. où l'on ne tient pas compte des phénomènes de charge d'espace.

Les figures 7 à 9 indiquent l'évolution de la largeur utile du faisceau en divers plans de la chambre d'ionisation pour $V_{0}=1000 \mathrm{~V}$ et deux valeurs extrêmes de $\Phi_{0}: \Phi_{0}=0,20 \mathrm{~V}$ et $\Phi_{0}=2 \mathrm{~V}$ (cette dernière valeur correspondant aussi à $\Phi_{0}=0,20 \mathrm{~V}$, $\left.V_{0}=100 \mathrm{~V}\right)$. Le plan II correspond à l'un des plans de section principale de la fente d'extraction des ions, le plan $\mathrm{A}_{3}$ est celui de l'électrode $\mathrm{A}_{3}$ (cf. fig. 1).

On voit qu'en II par exemple les dimensions transversales du faisceau sont de $0,35 \mathrm{~mm}$ à $V_{0}=1000 \mathrm{~V}$ et de $0,80 \mathrm{~mm}$ à $V_{0}=100 \mathrm{~V}$ pour des électrons d'énergie initiale $\Phi_{0}=0,20 \mathrm{eV}$.

La valeur $\sigma=V_{\mathrm{W}} / V_{0}=0,16$ qui a servi de base aux calculs correspond au relevé rhéographique dont nous disposions, le plus proche de la focalisation théorique optimale, mais en diffère légèrement puisque le faisceau correspondant à $\sigma=0,16$ converge en réalité un peu au-delà du plan de la trappe. Il s'ensuit que le faisceau à la focalisation optimale divergerait moins qu'indiqué sur les figures 7 à 9 .

Au niveau de $A_{3}$, la largeur du faisceau est de l'ordre de $0,4 \mathrm{~mm}$ pour $V_{0}=1000 \mathrm{~V}$ et de l'ordre de $1 \mathrm{~mm}$ pour $V_{0}=100 \mathrm{~V}$. Ce dernier point rejoint le résultat expérimental indiqué plus haut : avec une largeur de fente $A_{3}$ de $1,5 \mathrm{~mm}$, tous les électrons du faisceau sont collectés par la trappe sans rencontrer les parois de la chambre.

Dans la portion utile du faisceau électronique (comprise sensiblement entre les plans $\mathrm{P}_{1}$ et $\mathrm{P}_{3}$ ), l'épaisseur du faisceau reste inférieure à $0,4 \mathrm{~mm}$ pour $V_{0}=1000 \mathrm{~V}$. Elle passe seulement du simple au triple lorsqu'on divise la tension $V_{0}$ par un facteur 10 .

7. Conclusion. - L'étude expérimentale confirmée par le calcul théorique des trajectoires électroniques à travers le système accélérateur et dans la chambre d'ionisation, en l'absence d'induction magnétique auxiliaire de source, permet de conclure à un fonctionnement satisfaisant d'une telle source.

Sa stabilité est excellente, tant du point de vue du faisceau électronique que du point de vue du faisceau ionique extrait (dont les caractéristiques sont en cours d'étude).

Je remercie M. le Professeur Vauthier qui me proposa ce travail, effectué dans son labotatoire, ainsi que M. Septier, directeur scientifique au C.N.R.S., qui a bien voulu mettre à ma disposition les installations rhéographiques de l'Institut d'Électronique Fondamentale d'Orsay. Mes remerciements vont également à M. Boussard, maître-assistant, qui s'est occupé des relevés rhéographiques.

\section{BIBLIOGRAPHIE}

[1] Chantreau (J.), C. R. Acad. Sci, 1967, 265, 176-179.

[2] Vauthier (R.) et Chantreau (J.), C. R. Acad. Sci., 1960, 251, 1744-1748

[3] Chantreau (J.), C. R. Acad. Sci., 1961, 252, 695-697.
[4] BARnARD (G. P.), Modern Mass Spectrometry, The Institute of Physics, 1953, 2

[5] VAu'thier (R.), J. Physique Rad., 1952, 13, 107-108.

[6] Septier (A.), J. Physique Rad., 1954, 15, 573-581.

[7] Gans (R.), Z. Tech. Physik, 1937, 18, 41-48. 